\title{
Die Bedeutung der Geburtsschädigungen für die Entstehung des Schwachsinns und der Epilepsie im Kindesalter.
}

Von

Oberarzt Dr. Sehott, Stetten i. R.

In den Vorgeschichten schwachsinniger und epileptischer Kinder finden wir nicht zu selten Geburtsschädigungen aufgeführt. Nach Ansicht der Angehörigen sind dieselben schuld an dem später zutage tretenden Schwachsinn oder der Epilepsie. In ärztlichen Kreisen trifft man eine verschiedene Beurteilung. Die einschlägige Literatur weist wenige Bearbeitungen dieser Frage auf. Bei der allgemeinen Wichtigkeit der Frage ist es angebracht, einen Beitrag zu liefern. Aus Zweckmässigkeitsgründen werden Schwachsinn und Epilepsie getrennt behandelt werden.

\section{Schwachsinn.}

In 1100 Krankheitsgeschichten finden wir $150 \mathrm{mal}=13,63 \mathrm{pCt}$. Geburtsschädigungen erwähnt. In 30 Fällẹ $=2,81$ pCt. bilden diese Geburtsschädigungen die einzige Ursache.

Als Geburtsschädigungen sind genannt: Frühgeburt, schwere Geburt, Zangengeburt, Zwillingsgeburt, Drillingsgeburt, Sturzgeburt, Nabelblutung und scheintot geboren. In einzelnen Fällen finden wir ein Zusammentreffen mehrerer dieser Schädigungen. Es ist eine bekannte Erscheinung, dass bei der Entstehung des kindlichen Śchwachsinns mehrere ursächliche Beziehungen in Rechnung gestellt wèrden [Ziehen (1)]. Die Feststellung der ausschlaggebenden Ursache wird vielfach auf Schwierigkeiten stossen, nicht selten unmöglich sein, zumal wenn schon eine längere Zeit verflossen ist und ärztliche Beobachtungen nicht vorliegen. Je genauer man alles prüft, umsomehr gelangt man zu der Erkenntnis, dass die Beziehungen oft sehr verwickelt und verflochten sind. Es ergeben sich auch biologische Ausblicke, deren Verfolgung gewiss verlohnt, auf welche wir aber hier nicht näher eingehen können. 
Die folgende Zusammenstellung gibt einen Ueberblick über das Verhältnis der Geburtsschädigungen zu anderen ursächlichen Beziehungen des kindlichen Schwachsinns.

\begin{tabular}{|c|c|c|c|c|}
\hline & & Knaben & Mädchen & Summe \\
\hline $\begin{array}{c}\text { Geburtsschädigungen } \\
\qquad " \\
" \\
" \\
" \\
" \\
" \\
" \\
"\end{array}$ & 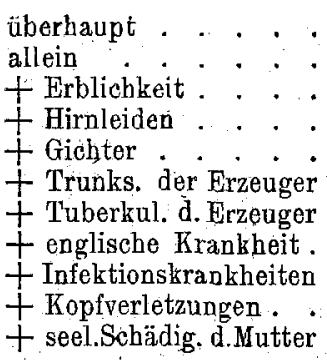 & $\begin{array}{l}13,71 \mathrm{pCt} . \\
3,14 " \\
4,43 " \\
1,14 " \\
0,85 " \\
0,71 " \\
0,71 " \\
0,28 " \\
0,42 " \\
0,28 \% \\
-\end{array}$ & $\begin{array}{c}13,50 \mathrm{pCt} \\
2,25 \% \\
3,50 " \\
1,75 \% \\
2,50 " \\
2,50 " \\
2,25 \% \\
1,25 " \\
1,00 " \\
0,25 \% \\
0,25 \%\end{array}$ & $\begin{array}{l}13,63 \mathrm{pCt} . \\
2,81 " \\
4,09 " \\
1,36 " \\
1,45 " \\
1,36 " \\
1,27 " \\
0,63 " \\
0,63 " \\
0,27 " \\
0,09 "\end{array}$ \\
\hline
\end{tabular}

Wir sehen also, dass in etwas über 13 pCt. Geburtsschädigungen genannt sind, welche aber nur in 2,81 pCt. als alleinige Ursache in Frage kommen: Am häufigsten treffen wir Geburtsschädigungen neben nervöser Belastung an, wobei der Schwachsinn fast die Hälfte der Belastung ausmacht, der mütterliche Anteil verhält sich zum väterlichen wie $1,6: 1$. Unter Hirnleiden sind begriffen: Mikrozephalie, Hydrozephalie und Hirnentzündung ohne nähere Angabe. $0 b$ es berechtigt ist, Gichter als gesonderte Krankheitsänsserung zu bewerten, kann fraglich erscheinen, zumal sie verschieden aufgefasst und beurteilt werden. Aus praktischen Gründen sind sie gesondert aufgeführt, wobei sich Verf. der Zweifelhaftigkeit dieser Eigenstellung bewusst ist. Sie zeigen eine erhöhte Gehirnerregbarkeit an und können Folgeerscheinung der Trunksucht der Erzeuger, angeborener Schädelmissbildung, der Rachitis u. a. sein. Dass bei der Trunksucht der Erzeuger den väterlichen Teil die Hauptlast trifft, ist unzweifelhaft. Zu den Geburtsschädigungen können noch Kopfverletzungen anderer Entstehung kommen; ausserdem ist in einzelnen Fällen die seelische Schädigung der Mutter während der Schwangerschalt noch zu dem kindlichen Schwachsinn in Beziehung gesetzt. .

Ein nennenswerter Unterschied der Geschlechter tritt bei den Gichtern, Trunksucht der Erzenger, Tuberkulose der Erzeuger und bei englischer Krankheit auf. Bei dẹ verhältnismässig kleinen Zahl der Untersuehten dürfte diesem Unterschied kein zu grosser Wert beigemessen werden. 
Ueber die Verteilung auf die einzelnen Geburtsschädigungen gibt nachstehende Aufstellung Aufschluss.

\begin{tabular}{|c|c|c|c|c|c|c|}
\hline \multicolumn{4}{|c|}{ Geburtsschädigungen } & \multicolumn{3}{|c|}{ Davon als alleinige Ursache } \\
\hline & Knaben & Mädchen & Summe & Knaben & Mädchen & Summe \\
\hline $\begin{array}{l}\text { Schwere Geburt. } \\
\text { Zangengeburt } \\
\text { Erühgeburt } \\
\text { Scheintod } \\
\text { Zwillingsgeburt. } \\
\text { Sturzgeburt . } \\
\text { Drillingsgeburt . } \\
\text { Nabelblutung . }\end{array}$ & $\begin{array}{l}3,14 \mathrm{pCt} . \\
3,57 " \\
3,42 " \\
2,57 " \\
0,71 " \\
0,28 " \\
0,14 " \\
0,14 "\end{array}$ & $\begin{array}{c}4,50 \mathrm{pCt} \\
3,25 " \\
1,75 " \\
2,25 " \\
2,25 \quad " \\
- \\
-\end{array}$ & $\begin{array}{l}3,63 \mathrm{pCt} . \\
3,45 " \\
2,81 " \\
2,45 \Rightarrow \\
1,27 " \\
0,18 \% \\
0,09 " \\
0,09 "\end{array}$ & $\begin{array}{c}1,00 \mathrm{pCt} . \\
0,85 " \\
0,43 " \\
0,85 " \\
0,28 \quad " \\
-\end{array}$ & $\begin{array}{l}1,00 \mathrm{pCt} . \\
0,50 \\
0,75= \\
- \\
- \\
-\end{array}$ & $\begin{array}{c}1,00 \mathrm{pCt} . \\
0,54 \quad " \\
0,45 \quad " \\
0,81 \quad " \\
0,18= \\
- \\
-\end{array}$ \\
\hline
\end{tabular}

Wir erkennen, dass die schwere Geburt an 1. Stelle steht, dann kommen in abnehmender Häufigkeit Zangengeburt, Frühgeburt, Scheintod u. a, Der Unterschied der Geschlechter dürfte bei dem geringen Umfange des Untersuchungsmaterials nioht von Bedeutung sein.

Ueber Geburtsschädigung und andere ursächliche Beziehungen gibt Nachfolgendes einen Ueberblick.

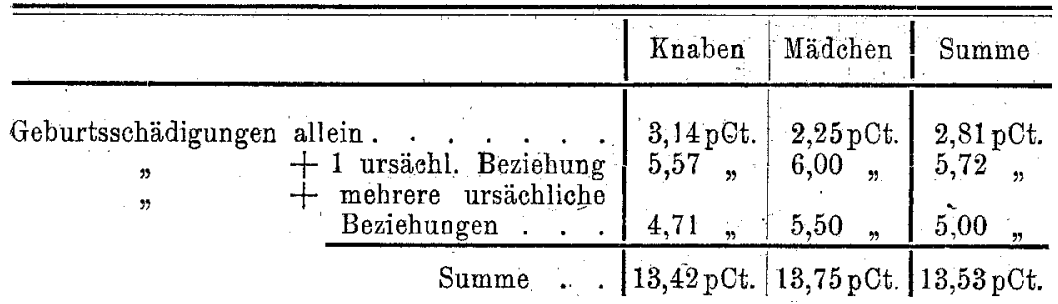

\section{Schwere Geburt.}

Es ist ausser Zweifel, dass eine schwere, langdauernde Creburt auf die Blutzufuhr des kindlichen Gehirns sehr nachterlig einwirken kann, insbesondere wenn wir noch auf Grund ererbter Anlage zu der Annahme berechtigt sind, dass dieses kindliche, Gehirn eine von Hause aus verminderte Widerstandsfähigkeit besitzt.

Schwere Geburt im Verein mit anderen ursächlichen Beziehungen.

\begin{tabular}{|c|c|c|c|c|}
\hline & Knaben & Mädchen. & Summe & Bemerkungen \\
\hline $\begin{array}{l}\text { Sehwere Geburt überhaupt } \\
\text { allein : } \\
\text { Nervöse Belastung } \\
\text { Trunksucht der Erzeuger } \\
\text { Gichter } \\
\text { Hirnentzündung . } \\
\text { Eltern Geschwisterkinder } \\
\text { Schwere Geb. + Scheintod }\end{array}$ & $\begin{array}{l}3,14 \mathrm{pCt} . \\
1,00 \cdots \\
1,14 " \\
0,28 " \\
0,28 \quad " \\
0,28 \% \\
0,28 \% \\
0,57 "\end{array}$ & $\begin{array}{l}4,50 \mathrm{pCt} . \\
1,00 ", \\
1,25 " \\
0,50 " \\
0,50 " \\
0,25 " \\
-\end{array}$ & $\begin{array}{l}3,63 \mathrm{pCt} . \\
1,00 \% \\
1,18 \% \\
0,36 \% \\
0,36 \% \\
0,27 \% \\
0,18 \% \\
0,36 "\end{array}$ & $\begin{array}{r}\text { Daruntor } 1 \text { Fall bei } \\
\text { [Seharlach }\end{array}$ \\
\hline
\end{tabular}




\begin{tabular}{|c|c|c|c|}
\hline & Knaben & Mädchen & Summe \\
\hline $\begin{array}{c}\text { Sehwere Geburt allein } \\
\Rightarrow \quad+1 \text { ursächliehe Beziehung } \\
\Rightarrow \quad+\text { mehrere ursächliche Be- } \\
\text { ziehungen . . . }\end{array}$ & $\begin{array}{l}1,00 \mathrm{pCt} \\
1,14 \% \\
1,00\end{array}$ & $\begin{array}{l}1,00 \mathrm{pCt} \\
2,25 \% \\
1,25 ;\end{array}$ & $\begin{array}{l}1,00 \mathrm{pCt} \\
1,54 \% \\
1,09 \%\end{array}$ \\
\hline
\end{tabular}

Die Mehrzahl der durch eine schwere Geburt in ihrer geistigen Entwicklung geschädigten Kinder war durch erbliche Belastung oder Trunksucht vermindert widerstandsfähig, nämlich $66 \mathrm{pCt}$; auch hier sehen wir sowohl von mütterlicher als auch vỏn väterlicher Seite den Schwachsinn entschieden überwiegen. Bei unseren Fällen wird in 80 pCt. der Schwachsinn ronseiten der Angehörigen als angeboren bezeichnet. Es ist auch hier schwer, den Tatsachen gerecht za werden, da die Möglichkeit nachträgliöher Gehirnerkrankungen jederzeit yegeben ist. Letztere werden nicht selten nicht nur von den Angehörigen, sondern : auch vom Arzt sei es verkannt, sei es falsch gedeutet.

Wenn in einem Falle im. 2. Jahre eine Schädelverletzung. ernsterer Art, in einem anderen Falle Hirnentzündung in demselben Alter oder Diphtheritis oder Scharlach mit Hirnentzündung aufgeführt sind und wenn erst nach diesen Vorfällen der Schwachsinn in Erscheinung getreten ist, so kann an dessen Entstehung, vorausgesetzt, dass die Beobachtung bezüglich des späteren Eintritts des Sch wachsinns richtig ist, der vorhergegangenen schweren Geburt nur in mittelbarer Weise eine Schuld beigemessen werden. Wir könnten uns dann vorstellen, dass durch die sehwere Geburt eine Minderwertigkeit des kindlichen Gehirns erzengt worden ist, auf deren Boden durch das neuerliche Vorkommnis der Schwachsinn sich ausgebildet hat.

Schon Vogt (2) hat vor der Ueberschätzung der Geburtsschädigung gewarnt. Lange dauernde Geburt ohne nennenswerte Zusammendrückung des Schädels stört den Blutumlauf des kindlichen Kopfes and damit des Gehirns auf längere Zeit.- Bei der ausserordentlichen Empfindlichkeit der Elemente des Nervensystems gegen Kreislaufstörungen ist nach Ziehen (1) eine "solche Entstehúngsursache des Schwachisinns sehr wohl verständlich. Wulff (3) hat bei 14 pCt. der Schwachsinnigen eine Schädigung des Kopfes. vor, während oder unmittelbar nach der Geburt festgestellt. Es sind besonders erstgeborene Kinder, bei welchen der Zusammenhang zwischen schwerer, langer Geburt und Schwachsinn deutlich zu erkennen ist: 
Virchow (4) hat schon 1851 angegeben, dass die besondere Ursache der Zerreissung and dadurch hervorgerufenen venösen Hirnblutung wahrscheinlich die beim Geburtsakt eintretende Uebereinanderschiebung der Schädelknochen ist.

B. Sachs (5) schreibt: „Es ist überraschend, wie viel Druck das Gehirn und der Schädel ohne Schaden aushalten können. Die lange Dauer der Geburt ist viel häufiger und in höherem Masse ätiologisch wirksam, als instrumentelle Entbindung".

\section{Zangengeburt.}

In 3,45 pCt. finden wir Zangengeburt erwähnt, in $0,54 \mathrm{pCt}$. als alleinige Ursache; ein nennenswerter Unterschied der Geschlechter ist nicht festzustellen.

Nachfolgende Zusammenstellung gibt einen genügenden Ueberblick.

Zangengeburt im Verein mit anderen ursächlichen Beziehungen.

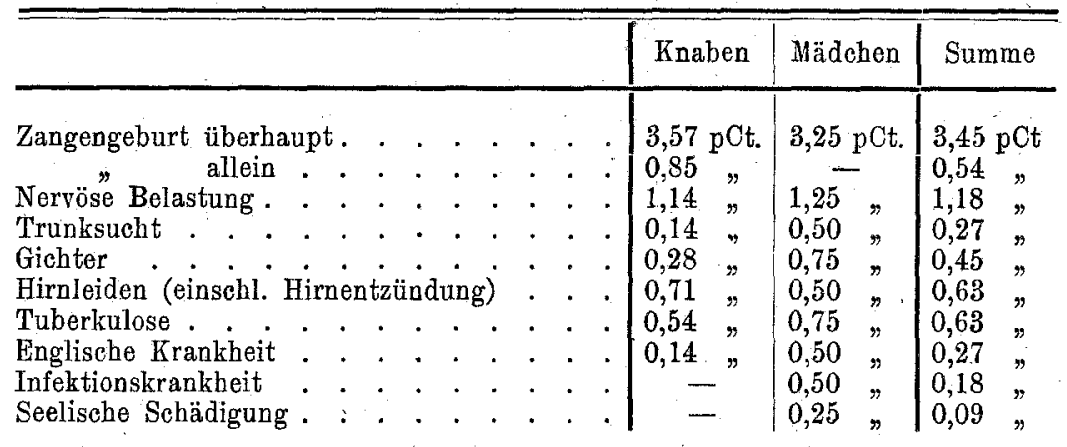

\begin{tabular}{|c|c|c|c|}
\hline & Knaben & Mädchen & Summe \\
\hline 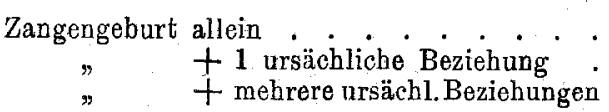 & $\begin{array}{l}0,85 \mathrm{pCt} . \\
1,14= \\
1,57\end{array}$ & $\begin{array}{l}1,50 \mathrm{pCt} \\
1,75\end{array}$ & $\begin{array}{l}0,54 \mathrm{pCt} . \\
1,27 \% \\
1,63 \%\end{array}$ \\
\hline
\end{tabular}

Wir sehen, dass auch bei der Zangengeburt die Hälfte der. Fälle erblich belastet ist, in $1 / 5$ besteht anderweitige Hirnschädigung. Für die Anschauung von B. Sachs (5), dass die lange Dauer der Geburt eine stärkere Schädigung des kindlichen Gehirnes bedinge, als die instrumentelle Entbindung, spricht das Verhältnis der schweren Geburt zur Zangengeburt in unserer Zusammenstellung, welches beinahe $2: 1$ beträgt.

Zweimal ist Zangengeburt mit Lähmung aufgeführt, so dass an einer schweren Hirnschädigung nicht gezweifelt werden kann. 
Zweimal findet sich ein Zusammentreffen von Zwillings- und Zangengeburt, einmal yon Früh- und Zangengeburt, einmal von Hydrozephalus und Zangengeburt. In allen diesen Fällen steht es ausser Frage, dass die Geburtsschädigung eine erhebliche war.

Bei Zangengeburten liegt nach Con.welaire (6) und Stewart (7) bemerkenswerter Weise die Blutung meist nicht am Orte der Zangeneinwirkung selbst, sondern im Hals mark oder im verlängerten Mark.

\section{Frühgeburt.}

Es ist leicht verständlich; dass Frühgeburt yon nachteiligem Einfluss auf die geistige Entwicklung sein kann. Bei der Frühgeburt entwickeln sich Seele und Verstand langsam, was mit der mangelhaften Ausbildung gewisser Zentren zusammenhängen mag. Ueberhaupt haben Frühgeborene eine erhöhte Neigung zu nervösen Erkrankungen. Besonders bei erstgeborenen kleineren Frühgeburten kommt es nach Wallich (8) häufig zu intrakraniellen Hämorrhagien and anderen Gehirnerkrankungen. Auch spielt die Frühgeburt in der Entstehung der Little'sehen Krankheit eine hervorragende Rolle. Frühgeburt kann in einzelnen Fällen nach Ziehen (1) die Ursache des Schwachsinns bilden. Die Vermutung von Ranke (9), dass bei Frühgeborenen auch ein normaler Akt der Geburt dadureh krankhafte Veränderungen hervorrufen kann, dass -die „unreifen" Kapillaren des Gehirns den erheblichen Kreislaufstörungen, welche die lösung des kindlichen. Körpers von der Nachgeburt begleiten, keinen genügenden Widerstand bieten, zerreissen und-so zu Blutungen Anlass geben, hat viel für sich. Bei erblicher Syphilis würde diese Widerstandsunfähigkeit der Kapillaren besonders gross sein, so dass auch bei Geburt zu normaler Zeit Blutungen sich einstellen können. Die Uebereinanderschiebung der Schädelknochen mit nachfolgenden venösen Blutungen kommt bei schnellen Geburten, daher auch bei Frühgeburten, leichter vor. Kleinere Blutungen machen meist keine Erscheinung [Ziehen(1)].' Auch Finkelsitein (10) hebt hervor, dass leichte, ohne Kunsthilfe erledigte Geburten zu submeningealen Blutungen führen können; insbesondere sind Frühgeburten dazu geneigt.

Frühgeburt ist in $2,81 \mathrm{pCt}$. sämtlicher t'älle genannt; als alleinige Ursache kommt sie in $0,45 \mathrm{pCt}$. in Frage. Fast in der Hälfte der Fälle erfolgte die. Frühgeburt im 7. Monat, in $1 / 4$ im 8. Monat. Beim Rest war kein Monat angegeben. Ein nennenswerter Unterschied der Geschlechter liess sich nicht feststellen. 
Frühgeburt im Verein mit anderen ursächlichen Beziehungen.

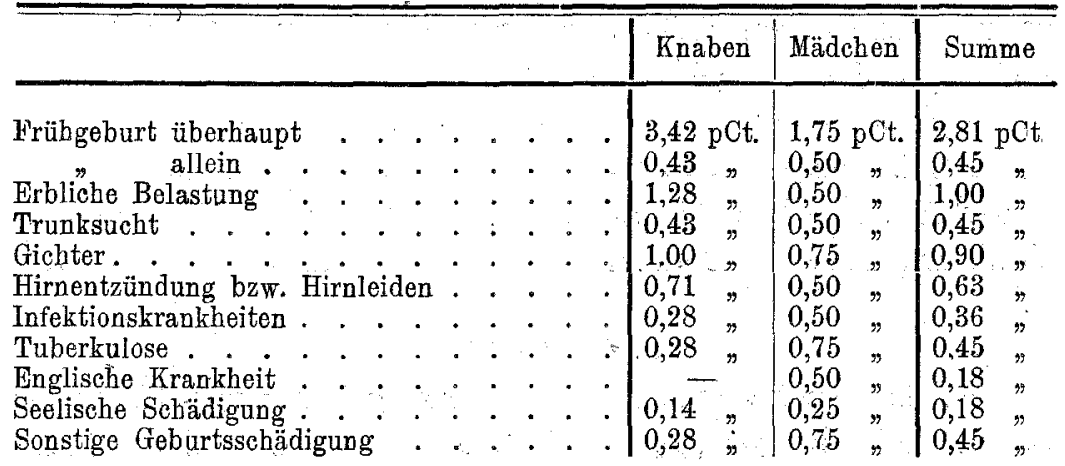

\begin{tabular}{|c|c|c|c|}
\hline & Knaben & Mädchen & Summe \\
\hline 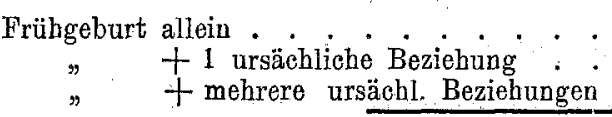 & $\begin{array}{l}0,43 \mathrm{pCt} . \\
1,57 \\
1,42 \%\end{array}$ & $\begin{array}{l}0,50 \mathrm{pCt} \\
0,50 \% \\
0,75 \%\end{array}$ & $\begin{array}{l}0,45 \mathrm{pCt} \\
1,18 \\
1,18\end{array}$ \\
\hline Zusammen & $3,42 \mathrm{pCt}$ & $1,75 \mathrm{pCt}$ & $2,81 \mathrm{pCt}$. \\
\hline
\end{tabular}

Annähernd die Hälfte der Fälle ist erblich belastet; in etwas weniger als $1 / 3$ liegt noch anderweitige Gehirnschädigung vor, in $1 / 6$ eine weitere Geburtsschädigung (Zwillingsgeburt, Zangengeburt, Scheintod). Die Tuberkulose der Erzeuger ist ebenfalls in $1 / 6$ erwähnt. Die Unterschiede der Geschlechter spielen bei dem kleinen Vergleichsmaterial keine nennenswerte Rolle.

\section{Scheintot geboren.}

Unzweifelhaft ist es nach Ziehen (1), dass Kinder, welche scheintot (asphyktisch) geboren wurden, öfter dem Schwachsinn verfallen, als normal geborene Kinder. Dieser asphyktische Zustand bedingt sehr wahrscheinlich an sich gleichfalls Schädigungen dès Gehirns. Bei der durch viele Tierversuche einwandfrei festgestellten Empfindlichkeit der Ganglienzellen des Zentralnervensystems selbst gegen kurzdauernde Absperrung der arteriellen Blutzufuhr ist ein solcher Zusammenhang wohl anzunehmen. Nach Rommel (11) werden zwei Formen von Asphyxie unterschieden: 1. Kongenitale Form - meist sub partu beginnend - Asphyxia cyanotica I. Grad, Asphyxia pallida II. Grad. 2. Erworbene Form - tritt erst nach der Geburt auf und wird meist Atelektase benannt.

Die Ursachen für die kongenitale Form, die eigentliche Asphyxie, sind: 
I. Von seiten des Kindes.

1. Kompression oder Umsehlingung der Nabelschnur.

2. Abnormer Hirndruck beim Fötus mit oder ohne Schädigung der Hirnsubstanz.

3. Frühgeburt von der 28 . Woche ab gerechnet.

4. Hämatombildung.

\section{Von seiten der Mutter.}

1. Ungenügende Zirkulation und Arterialisierung vonseiten der Mutter (besonders bei Herz- und Lungenerkrankungen).

2. Sinken des mütterlichen Blutdruckes bei Blutverlusten, Agonie, Tod der Mutter.

3. Wehenanomalien in der Austreibungsperiode, z. B. Tetanus uteri.

4. Yorzeitige Plazentarlösung, vorzeitigerBlasensprung, Placenta praevia, enges Beckën.

L. Seitz (12) hat $12 \mathrm{pCt}$. scheintote Geburten berechnet, davon tiefscheintot 3,5 pCt. Die Asphyxie betrifft nach Poppelt (13) hă̈ufiger Knaben als Mädchen. Die Sterblichkeit beläuft sich auf 20-30 pCt.

Die erworbene Asphyxie, anch Atelectasis pulmonum genannt, entsteht unter anderem durch Erkrankungen des Gehirns, besonders aueh Verletzungen desselben in der Gegend der Medulla oblongata dureh Geburtsschädigungen (Blutungen), auch kongenitalen Wasserkopf. Die Erscheinungen der schweren intermeningealen Géburtsblutungen weisen nicht immer auf das Gehirn hin. Es besteht vielmehr das Bild der schweren Asphyxie. Später jedoch treten hie und da Krämpfe auf oder es entwickelt sich auch ohne diese das Bild der spastischen Lähmung (zerebrale Kinderlähmung, Little'sche Krankheit). "In wieweit symptomlos verlaufende Geburtsblutungen 'später zu Epilepsie, Idiotie führen können, ist schwer zu entscheiden. Der Verdacht eines solchen Zusammenhangs besteht jedenfalls, wenn ohne erkennbare Ursachen bei mehrwöchigen Säuglingen sich Krämpfe einstellen und sich wiederholen" [Zappert (14)]. Runge (15) steht ebenfalls auf dem Standpunkt, dass scheintot geborene Kinder häufiger schwachsinnig werden als normal geborene.

Unter den aufgeführten Geburtsschädigungen steht die Asphyxie an vierter Stelle; sie ist beinahe ebenso häufig verzeichnet wie dic Frühgeburt. 
Asphyxie im Verein mit anderen ursächlichen Beziehungen.

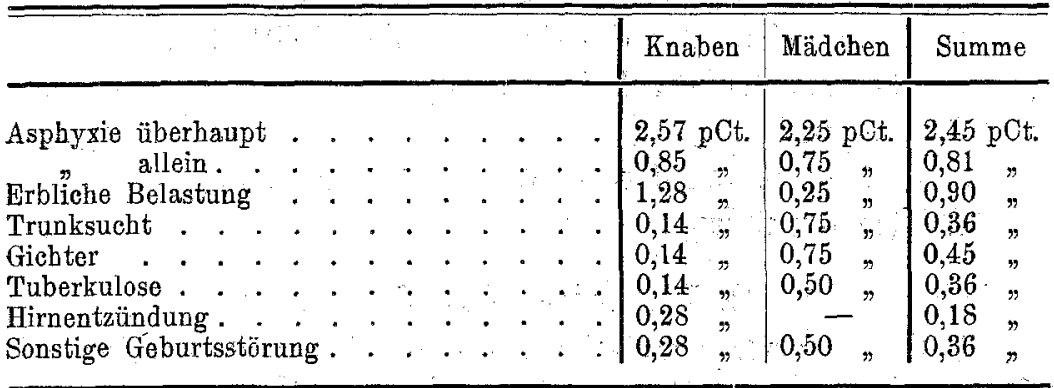

\begin{tabular}{|c|c|c|c|}
\hline & Knaben & Mädchen & Summe \\
\hline $\begin{array}{c}\text { Asphyxie allein } \\
\quad \Rightarrow \quad+1 \text { ursächliche Beziehung } \\
" \quad+\text { mehrere ursächliche Beziehungen }\end{array}$ & $\begin{array}{l}0,85 \text { pCt. } \\
1,28 \\
0,42 \%\end{array}$ & $\begin{array}{l}0,75 \mathrm{pCt} . \\
0,50, \\
1,00,\end{array}$ & $\begin{array}{l}0,81 \mathrm{pCt} . \\
1,00 \% \\
0,63:\end{array}$ \\
\hline Zusammen & $2,55 \mathrm{pCt}$. & $2,25 \mathrm{pCt}$. & $2,44 \mathrm{pCt}$. \\
\hline
\end{tabular}

Etwas mehr als ein Drittel der Fälle ist erblich belastet, wobei wieder der Schwachsinn stark überwiegt. Ein Ueberwiegen des männlichen Geschlechts hat sich bei unserem kleinen Zahlenmaterial micht nachweisen lassen.

\section{Zwillingsgeburt.}

Heimann (16) hat in seiner Statistik $10 \mathrm{mal}$ Zwillingsgeburt verzeichnet gefunden. Weicksel (17) hat 7 Fälle mitgeteilt, in denen sich angeborener Schwachsinn bei einem oder beiden Zwillingen fand. Er nimmt an, dass bei Zwillings- bzw. Mehrgeburten die Möglichkeit einer Schädigung des kindlichen Gehirns in der Entwicklung durch mangelhafte Ernährung gegeben sein kann. Infolgedessen ist bei Zwillingen eine erhöhte Disposition zu Schwachsinn vorhanden.

Die Zwillingsgeburt findet sich bei unserer Zusammenstellung in 1,27 pCt. aufgeführt.

Zwillingsgeburt im Verein mit anderen ursächlichen Beziehungen:

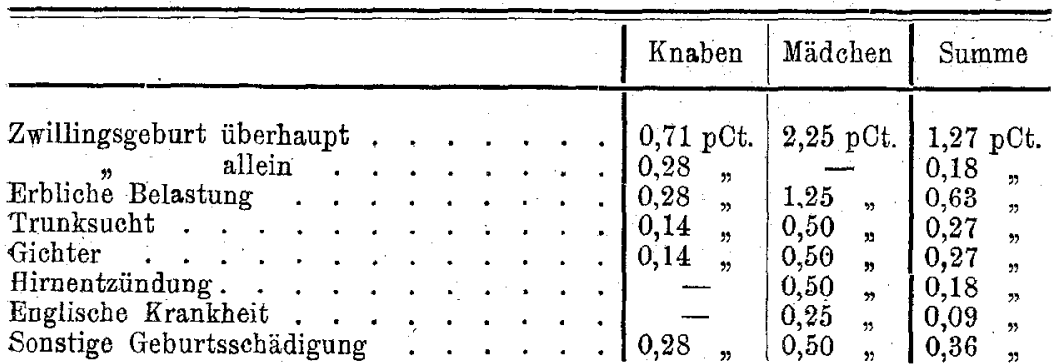




\begin{tabular}{|c|c|c|c|c|}
\hline & & Knaben & Mädchen & Summe \\
\hline \multirow[t]{2}{*}{$\begin{array}{c}\text { Zwillingsgeburt } \\
" \\
"\end{array}$} & $\begin{array}{l}\text { allein } \\
+1 \text { ursächliche Beziehung } \\
+ \text { mehrere ursächl Beziehung. }\end{array}$ & $\begin{array}{l}0,28 \mathrm{pCt} . \\
0,28 \% \\
0,14 \%\end{array}$ & $\begin{array}{l}1,25 \mathrm{pCt} \\
1,00\end{array}$ & $\begin{array}{l}0,18 \mathrm{pCt} . \\
0,63 \\
0,45\end{array}$ \\
\hline & Zusammen & 0,70 pCt. & $2,25 \mathrm{pCt}$ & $1,26 \mathrm{pCt}$. \\
\hline
\end{tabular}

In der Hälfte dẻr Fälle liegt erbliche Belastung vor; wobei auch hier der Schwachsinn vorherrscht. In einem Falle ist Zwillingszangengeburt mit rechtsseitiger Lähmung, in einem anderen Zwillingszangengeburt, in einem dritten Zwillings- und Frühgeburt aufgeführt. In $70 \mathrm{pCt}$. wird der Schwachsinn als angeboren bezeichnet, in den übrigen Fällen ist der Schwachsinn im ersten bzw. im zweiten Lebensjahre bemerkt worden. Zweimal soll der Schwachsinn nach Hirnentzündung, einmal nach Gichtern eingetreten sein.

\section{Sturzgeburt.}

Sturzgeburt finden wir nur bei Knaben und zwar in 0,18 pCt. erwähnt; als alleinige Ursache ist sie nicht aufgeführt. In dem einen Falle war die Mutter schwachsinnig, in dem anderen bestand von mütterlicher Seite erbliche Belastung im Sinne der Geisteskrankheit. $O b$ das Kind bei der Sturzgeburt eine nennenswerte Kopfverletzung erlitten hat, ist nicht nachgewiesen. Es könnte auch hier, ähnlich wie bei Frühgeburten, zu submeningealen Blutungen kommen.

\section{Drillingsgeburt.}

Für die Drillingsgeburt gilt im wesentlichen das für die Zwillingsgeburt Gesagte. Bei unserer Durchsicht kommt sie nur mit $0,09 \mathrm{pCt}$. in Betracht, ausserdem nicht als alleinige Ursache. In dem betreffenden Fall sind die beiden anderen Drillingsbrüder während bzw. kurz nach der Geburt gestorben. Der Knabe erlitt mit $3 / 4$ Jahren eine schwere Kopfverletzung, von welcher jetzt noch Spuren am knöchernen Schädel nachweisbar sind. Die verlangsamte geistige Entwicklung machte sich im 2. Lebensjahre geltend. Wenn wir im vorliegenden Falle der Drillingsgeburt als solcher überhaupt eine Bedeutung für die Entstehung des kindlichen Schwachsinns beimessen wollen, so kann das nur mittelbar geschehen in der Annahme, dass eben die Drillingsgeburt ein schwächlicheres Kind zeitigt als eine Erstlingsgeburt, und dass infolgedessen die Einwirkung der Kopfverletzung sich unheilvoller geltend.ge- 
macht hat. Diese Voraussetzung trifft jedoch nur unvollkommen $\mathrm{zu}$, da wir es mit einer so erheblichen Kopfverletzung zu tun gehabt haben, dass diese als solche die geistige Schädigung genügend erklärt.

\section{Nabelblutung.}

Schliesslich ist noch in 0,09.pCt. der Nabelblutung gedacht, aber auch nicht als alleiniger Ursache. Im Gegenteil finden wir gerade in diesem Falle ein Zusammentreffen mehrerer ursächlicher Beziehungen: die Eltern sind Geschwisterkinder, bei der Geburt Nabelblutung; dann englische Krankheit, Scharlach, perniziöse Anämie, an welcher der Knabe später klinisch nachgewiesen gestorben ist. Es handelt sich demnach um eine angeborene Blutkrankheit, welcher die Nabelblutung ihre Entstehung verdankt und auf deren Boden, nämlich der Blutkrankheit, auch die Verlangsamung der geistigen Entwicklung erwachsen ist.

\section{Zusammenfassung.}

Wenn wir die aufgeführten Geburtsschädigungen überblicken, so erkennen wir, dass sie als allëinige Ursache des Schwachsinns wenig ins Gewicht fallen. Sie stellen in einer Reihe von Fällen eine Ausdrucksform der mangelhaften Veranlagung der Mutter dar. Dass gerade eine Geburtsschädigung als Ursache des kindlichen Schwachsinns von den Angehörigen in Anspruch genommen wird, ist leichtverständlich, da dieses Ereignis so sehr in die Augen springt und Eindruck macht. Bei kritischer Beleuchtung allerdings zeigt es sich, dass die Geburtsschädigungen als solche mit annähernd $3 \mathrm{pCt}$. als alleiniger Ursache im Vergleiche zu den anderen ursächlichen Beziehungen des kindlichen Schwachsinns wenig zu bedeuten haben. Im Verein mit anderen ursächlichen Beziehungen stellen sie bei gegen $14 \mathrm{pCt}$. ein beachtenswertes Moment dar. Wir müssen uns also einerseits vor Ueberschätzung dieser Ursache hüten, andererseits bei Geburtsschädigungen die sonstigen Umstände des kindlichen Werdens und Abstammens ins Auge fassen, um uns bezüglich des weiteren Verlaufes den Blick nicht trüben zu lassen und um uns vor unliebsamen Ueberraschungen nach Möglichkeit zu schützen. Je besser die Bedingungen der Schwangerschaft, je günstiger die Voraussetzungen der Geburt sind, um so eher werden wir in der Lage sein, eine Geburtssehädigung hintanzuhalten. 
Nach Zappert (14) ist die Annahme einer Geburtssehädigung in erster Linie dann berechtigt, wenn eine sehwere Geburt oder eine Asphyxie vorhanden gewesen war oder wenn bald nach der Geburt sich Krämpfe eingestellt haben. Aber auch ohne Geburtsschwierigkeit, namentlich bei sehr rasch ablaufenden Geburten, sind intrazerebrale Blutungen möglich; vielleicht sind auf diese Weise die nach Frühgeburten vorkommenden Zerebrallähmungen zu erklären. Mondio (18) ist der Ansicht, dass bei Entstehung dieser Lähmungen der Geburtsschädigung nur die Rolle einer Gelegenheitsursache zuzuerkennen ist. „Das. Hauptmoment liegt im neuropathischen und krankhaften Gehirnzustande der Mutter, hauptsächlich auch während der Sehwangersehaft ${ }^{u}$. Wir finden in etwas mehr' als einem Drittel erbliche nervöse Belastung verzeichnet, wobei der mütterliche Anteil beinahe doppelt so gross ist als der vääterliche. Der Schwachsinn macht die Hälfte dieser Fälle aus; Geisteskrankheit, Epilepsie und Nervosität je $1 / 6$.

Auf die Trunksucht entfällt $1 / 9$ der Geschädigten. In 4,6 pCt. sind die Eltern Geschwisterkinder. Wenn wir alle diese 3 Beziehungen zusammenfassen, so ergibt sich eine Gesamterblichkeit von $50 \mathrm{pCt}$., während Wulff (3) eine Gesamterblichkeit von $53,3 \mathrm{pCt}$. berechnet hat. Er hat in $13,8 \mathrm{pCt}$. Geburtsschädigungen des Kopfes bei schwachsinnigen Kindern gefunden und äussert sich darüber wie folgt: „Anamnestisch wird nun ofter als Ursache eine einfache, schwere oder lange dauernde Geburt angegeben, doch dürfen wir meines Erachtens diese nur dann berücksichtigen, wenn sie ärztlicherseits als schwere, den kindlichen Schädel entschieden beeinträchtigende Geburt bestătigt wird". Nach ihm ist der Prozentsatz der Belasteten so hoch, dass man geneigt wird, anzunehmen, dass jede das Gehirn treffende schädliche Einwirkung, die bei nicht belasteten Individuen, vielleicht ohne irgend welche Spur zu hinterlassen, vorübergeht, bei erblich belasteten Menschen ausserordentlich leicht dauernde, zum Idiotismus führende Störungen hervorruft. Die pathologischen, meistens chronisch verlaufenden Prozesse, die als Folgen der betreffenden Schädigungen des kindlichen Kopfes anzusehen sind, sind natürlich nicht einheitlicher Natur; sie können als kongestive, als entzündliche, als. sonstige Ernährungsstörungen des Gehirns, der Hirnhäute und des Schädels (vorzeitige Synostosen) auftreten und bedingen im allgemeinen und der Hauptsache nach eine Funktionsstörung der Hirnrinde. Vor- 
zeitige Synostosen am Schädel sind nach. Wulff (3). sehr häufig, 40 pCt.

Idiotie scheint nach Savage (19) nicht besonders häufig auf künstliche Entbindung zu folgen; es ist wohl sicher, dass Verletzungen kurz vor oder nach der Geburt Blödsinn bewirken können, ebenso aber auch, dass solche in den ersten Kinderjahren bedeutend häufiger sind. Bei der Geburt können in seltenen Fällen Beeinträchtigungen des Kopfes schädlich werden [Griesinger (20)]. Während der Geburt können nach v. Krafft-Ebing (21) traumatische Schädlichkeiten auf das kindliche Gehirn einwirken, die zu Idiọtismus führen; in der überwiegenden Mehrzahl der Fälle konmen aber die Schädlichkeiten erst nach der Geburt, in den Jahren der Kindheit zur Geltung.

Beim Geburtsvorgang selbst kann nach Emminghaus (22) Idiotie noch begründet werden durch Schädlichkeiten, welchè den Kopf treffen. Mitchell (23), Koch (24), Bramwell (25), Winkler (26) u. Bollaan u. a. haben hierher gehörige Fälle beschrieben. Fletcher (27)-Beach fand unter 810 Fällen von Idiotie 4,3 pCt., die infolge der Anwendung der Zange, und 26,6 pCt., welche durch schwierigen, lange dauernden Geburtsverlauf die geistige Störung erlitten. W. Koenig (28) fand Frühgeburt in 3,8 pCt., schwere bzw. asphyktische Geburt in 10 pCt. bei einfacher Idiotie, bei zerebraler Kinderlähmung $10 \mathrm{pCt}$. bzw. 11,4 pCt. Küntzel (38) führt 0,9 pCt. seiner Fälle von Tdiotie auf die Zange, 4,5 pCt. auf schwere Geburten ohne Kunsthilfe zurück.

Klotz (29) gibt eine Uebersicht über die verschiedenen, sehr auseinandergehenden Ansichten, die in der Literatur über die Bedeutung der Geburtsschädigungen für die körperliche und geistige Entwicklung, insbesondere auch für die Entstehung der Little'schen Krankheit, niedergelegt sind. Bei 144 Idioten und Epileptikern fand Klotz (29) $19 \mathrm{mal}$ abnorme Geburt als Ursache angegeben; davon war bei 11 Kindern (7,6 pCt.) irgendwelches andere ätiologische oder prädisponierende Moment nicht vorhanden. Auffälliger Weise war bei keinem der 18 Fälle von Little'scher Krankheit, die unter den 144 Kindern vorhanden waren, Geburtsschädigung oder Frühgeburt als Ursache nachzuweisen.

H. Vogt (2) hat 1100 Fälle von Idiotie auf den Zusammenhang von Geburtsanòmalien hin geprüft. $78 \mathrm{mal}$ hat er eine schwere Geburtsstörung gefunden und zwar $47 \mathrm{mal}$ die Angabe 
schwere Geburt, $11 \mathrm{mal}$ asphyktische Geburt und $20 \mathrm{mal}$ Zangengeburt. Alleinige Geburtsschädigung in der Anamnese wurde nur 17 mal gefunden. Vogt (2) hat demnach 1,54 pCt. gefunden gegenüber 2,81 pCt. unserer Zusammenstellung.

Aus der Statistik ergibt sich, was schon Massalongo (30), Runge (15), Blind (31) u. a. vermutet hatten, dass der Einfluss der erschwerten Geburt ganz erheblich überschatzt worden ist. In den Fällen mit asphyktischer und einfach erschwerter Geburt sind wir nach Vogt (2) beim Vorliegen noch anderer, namentlich schwerwiegender ätiologiseher Homente, so, z. B. schwerer Familiendegeneration, schwererem Alkoholismus der Eltern, Lues usw. berechtigt, die Geburtsștorung mit der Krankheit in einen einfach ursächlichen Zusammenhang zu-bringen. Anders steht es nach Vogt (2) bei der Zangengeburt: namentlich beitypischen Little-Fällen müssen andere ursächliche Faktoren in der Schätzung, hierbei zurücktreten, einfach erfahrungsgemäss! „Die Zange selbst ist auch ein schwerer Eingriff; die protrahierte Geburt gehört aber doch bis zu gewissem Grade in den Bereich des Physiologischen".

Was schliesslich die pathologische Anatomie der hierher gehörigen Fälle betrifft, so ist folgendes zu sagen. Intrakranielle Blutungen sind entweder Folgen von Geburtsschädigungen oder von später einwirkenden Schädlichkeiten. Unter den Ursachen der Hirnblutungen nach der Geburt sind Verletzungen, Keuchhusten, Purpura, schwere Atrophie, sowie anderweitige Hirnerkrankungen, so namentlich Sinusthrombose bemerkenswert. Die während der Geburt entstehende Blutung in die Gehirnhäute besitzt nach Zappert (14) eine noch immer zu wenig gewürdigte Bedeutung. "Untersucht man wahllos eine grössere Anzahl von Neugeborenen am Sektionstisch, so ist man überrascht über die Häufigkeit von Blutaustritten innerhalb der Schädelhöhle. Sicherlich wird das Blut in der Mehrzahl der Fälle bei sonst gesunden Neugeborenen resorbiert, ohne klinische Erscheinungen hervorzurufen. Aber in einer nicht unbeträchtlichen Minderzahl von Fällen besitzt die Blutung eine derartige Ausdehnung, dass sie das Weiterleben des Kindes unmöglich machen musste. Zwischen diesen beiden Extremen müssen jedenfalls eine grosse Reihe von Zwischenstufen existieren, deren Erkennung uns schwer möglich ist, die aber für das Zustandekommen späterer zerebraler Symptome von grosser Bedeutung sein können". Die richtige Würdigung der submenin- 
gealen Blutung wird um so schwerer, als die Ursache derselben durchaus nicht immer klarliegt. In der Mehrzahl der Fälle wird allerdings eine schwere, protrahierte. Geburt mit starker Verschiebung der Kopfknochen [Kundrat (32)] das. Entstehen von Stauungen und Gefässzerreissungen innerhalb des Schädels verständlich erscheinen lassen.

Der Sitz der Blutung ist nach Zappert (14) in der Mehrzahl der Fälle die Höhe des Scheitels, etwa die Gegend der beiden Parazentralläppchen. An dieser Stelle findet man starke Auflagerungen geronnenen Blutes. Die Blutungen können sich auf die eine oder beide Konvexitäten der Grosshirnhemisphären [Sarah Mac. Nutt (33)], seltener auf die Hirnbasis, das Kleinhirn erstrecken. Die unterhalb der Blutansammlung liegenden Teile der Hirnrinde sind komprimiert, blutig imbibiert. Als Endprodukte sind bei : überlebenden Kindern Porenzephalie, Hydrocephalus externus, lokale Meningoenzephalitis: u. a. zu gewärtigen.

H. Vogt (2) hat bei Sektionen gefunden: bald zerstreute mikroskopische Veränderungen, bald grössere Herde, und zwar teils Blutungen, teils Erweichungen, teils Enzephalitiden; namentlich an der Basis findet man sie nicht selten gehäuft.

W. Beuthin (34) hat 10. Fälle untersucht, bei welchen während oder bald nach der Geburt der Tod des Kindes, in den letzten Fällen bei zunächst zu beobachtender guter Herztätigkeit, unter den für intrakranielle Blutungen charakteristischen Zeichen auftrat, und in welchen die Sektion mit Sicherheit als Ursache derselben eine Zerreissung des Tentoriums ergab.

Nach Beuthin (34) bedürfen die Tentoriumzerreissungen keiner grossen Gewaltanwendung. Es könne schon ein forcierter Dammschutz in der Austreibungsperiode diese herbeiführen. Im allgemeinen berechnet Beuthin (34) nach den Sektionén eine Sterblichkeit von $10 \mathrm{pCt}$. an Tentoriumzerreissung.

Beneke (35) ist auf Grund seiner Untersuchungen und Beobachtungen za der Ueberzeugung gekommen, dass sehr viel häufiger als örtliche Zertrümmerungen die Blutungen der Hirnhäute und besonders diejenigen im Subduralraum sind. Sitzen dieselben im Gebiete der Konvexität der Grosshirnhemisphären, so entstehen sie wohl zweifellos meistens durch die von Kundrat (32) studierte Zerreissung der den Subduralraum dnrchsetzenden kleinen Piavenen, namentlich in der Nähe des Sinus longitudinalis sup.; diese Zerreissung ist natürlich die Folge einer jähen Verschiebung der Dura, namentlich im Nahtgebiete, vor allem wohl einer Unter- 
schiebung der Parietalbeine untereinander. Nach Beneke (35) sind die Blutungen im Gebiete des Tentorium cerebelli die häufigsten. Sie umspülen die untere Fläche des Okzipitalhirns und von da, in ganz glatter Ebene über den Kamm des Felsenbeins vordringend, die basale Fläche des Temporallappens; oft können sie bis an die vordere Spitze der Schläfenlappen verfolgt werden, aber auch im grossen Medianspalt oder lateral an der Konvexität des Okzipitalhirns aufsteigen. Auch die oberé Fläche des Kleinhirns zeigt nicht selten diese Blutung. Nach Beneke (35) ist diese Blutungsform als Ursache des asphyktischen Kindes oder mehr weniger schwerer Hirnerkrankungen ungemein wichtig.

Die Zerreissungen des Tentoriums, welches aus zwei Blättern, einem oberen von der Dura des Grosshirns ausstrahlenden und einem unteren, der Kleinhirndura entsprechenden, besteht, betreffen vorwiegend das obere Blatt und nicht selten den freien Rand. Schon aus dieser Tatsache ist nach Beneke (35) ihre genetische Beziehung zu der Spannung der Grosshirndura zu erkennen. Die Form der Löcher ist mannigfach, entsprieht aber allermeistens einem Auseinanderweichen der scharf gerichteten Faserzüge in ihrer Längsrichtung, wodurch ein mehr weniger breiter, zur Faserrichtung senkrecht stehender Rissdefekt entsteht. Eine jähe oder bisweilen auch eine anhaltende Kompression des Schädels von Schläfe zu Schläfe während des Geburtsaktes in irgend einer Phase desselben veranlasst demnach die Zerreissung bzw. das Auseinanderweichen der Tentoriumstrahlung der Falx. Die gröbsten Tentoriumzerreissungen finden sich bei Kindern, welche im Anschluss an eine schwere Geburt tot geboren worden oder alsbald asphyktisch zugrunde gegangen sind; aber auch bei ganz normalen Geburten, ja bei ausgesprochen leichten, raschen Geburten und Frühgeburten konnte eine Zerreissung nachgewiesen werden. Die Möglichkeit, dass auf diesem Wege bei normaler Geburt der Tod des Kindes durch "Asphyxie" erfolgen kann, ist klinisch wie auch forensisch bedeutungsvoll. Der Grad der Folgeerscheinungen hängt von der Ausdehnung der Blutung ab, da die Tentoriumzerreissung an sich wenig zu bedeuten hat. Starke Blutungen können zum Druck auf das verlängerte Mark und damit zum asphyktischen Tode führen; geringere unter Umständen zu Druckerscheinungen und hierdurch zu vorübergehenden oder vielleicht einmal za dauernden Schädigungen der Okzipital- und Schläfenhirnrinde, wodurch tage- und wochenlang bestehende un- 
klare Hirnerscheinungen in manchen Eällen ihre Erklärung finden möchten, ebenso wie weiterhin sich anschliessende Zustände von Epilepsie, Idiotie u. a.

Stolper (36) sezierte einen schweren Fall von Porenzephalie eines Kindes, das nach 2 tägiger Wehendauer mittels Zange bei verengtem Becken der Mutter geboren war.

L. Meyer (37) führt gewisse Schädeldeformitäten bei originär Schwachsinnigen auf traumatische Einflüsse während der Geburt zurück.

Winkler und Bollaan (26) haben bei 10 zur Sektion gekommenen Idioten Eindruckstellen am Schädel vorgefunden, die sie auf ein Trauma während der Geburt zurückführten. Ob auch durch die Asphyxie als solche erheblichere Blutungen entstehen können, ist nach Ziehen (1) zweifelhaft. Die Schultze'schen Schwingungen hat man für das Auftreten eigentümlicher spaltförmiger Blutungen yerantwortlich machen wollen. Nach dem heutigen Stande unseres Wissens liegt die Vermutung nahe, dass es sich auch hier um Tentoriumszerreissungen gehandelt hat.

Wir sehen aus dem Vorstehenden, dass einwandfreje pathologisch-anatomische Befunde vorliegen, welche für die klinische Auffassung der Störungen von einschneidender Bedeutung sind.

\section{Benütate Literatur.}

1. Ziehen, Handb. d. Nervenkrankh. im Kindesalter.

2. H. Vogt, Arch. f. Kinderheilk. Bd. 48. H. 5.

3. W ulff, Allg. Zeitschr. f. Psych. 1893. Bd. 49 u. Zeitschr. f. Beh. Schwachs. 1889. H. 5.

4. Virchow, Verh. d. Würzburger phys. mediz. Ges. 1850. Bd. 2. S. 11.

5. B. Sachs, Lehrb. d. Nervenkrankh. d. Kindesalters. 1897.

6. Convelaire, Compt. rend. de la soc. de biol. 28. III. 1903. Ref.

7. Stewart, New York med. journ. 10. I. 1903. Ref.

8. Wallich, Zitiert nach Rommel (11), Handb. d. Kinderheilk. v. Pfaundler u. Schlossmann. Bd. 1.

9. Ranke, Vortrag. Naturforscherversamml. Strassburg. 1885.

10. Finkelstein, Berl. Klinik. 168.

11. Rommel, Handb. d. Kinderheilk. v. Pfaundler u. Sohlossmann. Bd.1.

12. L. Seitz, Der Scheintod des Neugeborenen, Handb. d. Geburtsh, von v. Winckel. 1907.

13. Poppeit, Zitiert nach Rommel (11).

14. Zappert, Handb. d. Kinderheilk. v. Pfaundler u. Schlossmann. Bd. 4. S. $248 \mathrm{ff}$.

15. Runge, Die Krankheiten der ersten Lebenstage. Stattgart 1906.

16. Heimann, Allg. Zeitscht. f. Psyob. Bd. 60. S. $443 \mathrm{ff}$.

17. Weicksel, Zeitschr. f. d. ges. Neurol. u. Psych. Bd. 15.

18. Mondio, Gaz. degli osped. 1901. Nr. 21. 
19. Savage, Geisteskrankheiten. 1887.

20. Griesinger, Path: u. Ther. 1883.

21. . Krafft-Ebing, Lehrb. 1883.

22. Emminghaus, Die psychischen Störungen im Kindesalter,

23. Mitchell, Transact. of the obstet, soc. 1862.

24. Ko ch (Kopenhagen). Ref. Neurol. Zentralbl. 1887:

25. Bramwell, The brit. med. journ. Ref. Neurol. Zentralbl. 1887.

26. Winkler u. Bollaan. Ref. Neurol. Zentralbl. 1889.

27. Fletcher-Beach, The Lancet 1889. Ref. Dentsche Mèd.-Ztg. 1889.

28. W. Koenig, Allg. Zeitschr.f. Psych. 1904. Bd. 61 u. Zeitschr. f. Nervenbeilk. Bd. 13.

29. Klotz, Zeitschr. f. d. ges. Neurol. u. Psych. Ref. Bd. 8. 1913 u. Monatsschrift f. Kinderheilk. Ref. Bd. 11. H. 12.

30. Massalongo, Ueber zerebrale Diplegien im Kindèsalter. Wiener med. Blätter. 1898. Nr. 7.

31. Blind, Inaug.-Diss., Würzburg 1910.

32. Kundrat, Porenzephalie. Graz 1882.

33. Sarah Mac Nutt, Zitiert nach Zappert.

34. W. Beuthin, Monatsschr. f. Geburtsh. Bd. 36. H. 3.

35. Beneke, Münchener med. Wochensohr. 1910. Nr, 4Г.

36. Stolper, Vierteljahrsschr. f. ger. Med. a. öfentl. Sanitätswesen. 3. Folge. Bd. 13.

37. I. Meyor, Zitiert naeh Stolper (36).

38. Küntzel, Zitiert nach Stolper (36).

\section{Epilepsie.}

Für die Entstehung der kindlichen Epilepsie kommen, in $1,09 \mathrm{pCt}$. Geburtssehädigungen als alleinige Ursache in betracht; in $8,54 \mathrm{pCt}$. werden solche erwähnt. Ein auffälliger Unterschied der Geschlechter ist nicht zutage getreten, zumal bei der Zahl von 1100 epileptischen Kindern die Vergleichszahlen zu gering sind.

im Verein mit anderen ursächlichen Beziehungen.

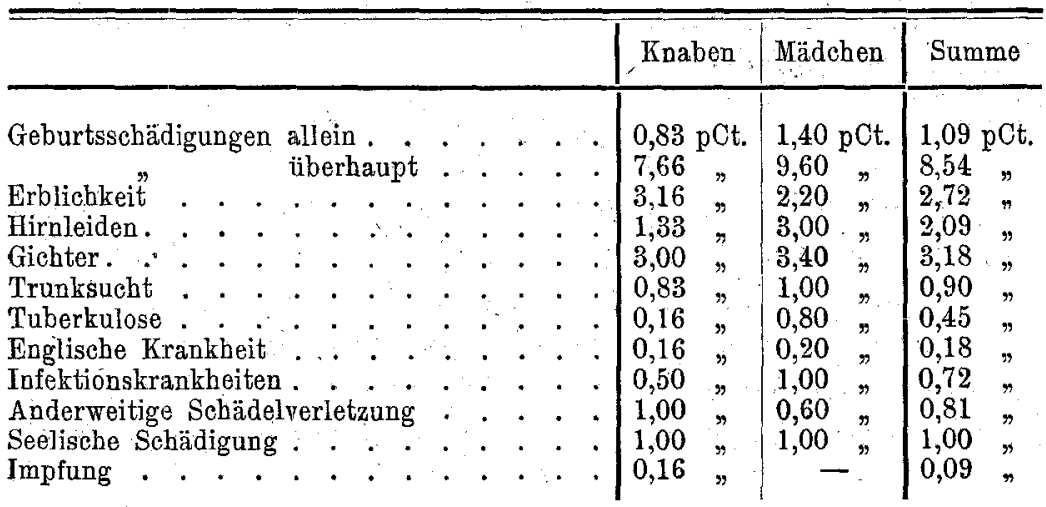




\begin{tabular}{|c|c|c|c|}
\hline & Knaben & Mädehen & Summe: \\
\hline 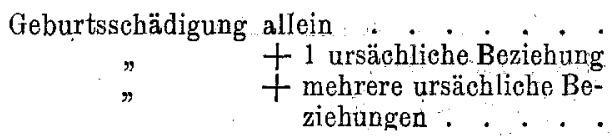 & $\begin{array}{l}0,83 \mathrm{pCt} . \\
2,83 \% \\
4,00\end{array}$ & $\begin{array}{l}1,40 \mathrm{pCt} . \\
4,40 \% \\
3,80\end{array}$ & $\begin{array}{l}1,09 \mathrm{pCt} . \\
3,54 \\
3,90\end{array}$ \\
\hline
\end{tabular}

Wir erkennen aus dieser Zusammenstellung, dass erbliche Belastung mit Einschluss der Trunksucht fast die Hälfte der Fälle betrifft; dann folgen in abnehmender Häufigkeit: Gichter, Hirnleiden; seelische Schädigung, anderweitige Schädelverletzung und die anderen ursächlichen Beziehungen. Bemerkenswert ist, dass in fast der Hälfte der Fälle neben der Geburtsschädigung noch mehrere ursächliche Beziehungen in Frage kommen (genau in 45,74 pCt.). Von Geburtsschädigungen finden wir erwähnt: Schwere Geburt; Zangengeburt, Frühgeburt, Zwillingsgeburt, scheintot geboren and Nabelblutungen.

\begin{tabular}{|c|c|c|c|c|c|c|}
\hline \multicolumn{4}{|c|}{ Geburtsschädıgungen } & \multicolumn{3}{|c|}{ Davon als alleinige Ursache } \\
\hline & Knaben & Mädchen & Summe & Knaben. & Mädchen & Summe \\
\hline $\begin{array}{l}\text { Zangengeburt } \\
\text { Schwere Geburt } \\
\text { Scheintot gebor. } \\
\text { Frühgeburt . } \\
\text { Zwilingsgeburt } \\
\text { Nabelblutung }\end{array}$ & $\begin{array}{l}2,16 \mathrm{pCt} . \\
2,16 \% \\
1,33 " \\
1,00 " \\
1,00 "\end{array}$ & $\begin{array}{l}3,00 \mathrm{pCt} . \\
2,80 " \\
1,60 " \\
1,00 " \\
0,80 " \\
0,20 "\end{array}$ & $\begin{array}{l}2,54 \mathrm{pCt} \\
2,45 " \\
1,45 " \\
1,00 " \\
0 ; 90 " \\
0,09 "\end{array}$ & $\begin{array}{c}0,16 \mathrm{pCt} . \\
0,33= \\
0,16= \\
- \\
-\end{array}$ & $\begin{array}{c}0,20 \mathrm{pCt} \\
0,40= \\
- \\
-\end{array}$ & $\begin{array}{c}0,09 \mathrm{pCt} . \\
0,27 " \\
0,27 " \\
- \\
-\end{array}$ \\
\hline
\end{tabular}

Wir sehen aus dieser Zusammenstellung, dass die Zangengeburt vorangeht, dann kommt die schwere Geburt, an dritter Stelle folgt die scheintote Géburt, weiterhin die anderen. Ein nennenswerter Unterschied der Geschlechter konnte nicht erwiesen werden. Als alleinige Ursache kommt keine der genannten Geburtsstörungen nennenswert in betracht. Im Verein mit anderen ursächlichen Beziehungen entfallen auf Zangengeburt und schwere Geburt je $21 / 2$ pCt.

\section{Zangengeburt.}

Es ist naheliegend, bei der Zangengeburt grobe Gehirnschädigungen anzunehmen, welche die Veranlassung zur Entstehung der Epilepsie geben. In der Mehrzahl der Fälle wird der Zusammenhang nicht so klar zutage liegen; nur in einem unserer Fälle ist diese Vermutung berechtigt, da schon bald nach der Zangengeburt rechtsseitige Zuckungen und später allgemeine Krämpfe 
aufgetreten sein sollen. In fast allen Fällen finden wir ein Zusammentreffen mehrerer ursächlicher Beziehungen, welches einen genauen Entscheid vielfach schwer macht. Die nachstehende Uebersicht zeigt dieses: Verhältnis.

Zangengeburt im Verein mit anderen ursächliehen Beziehungep.

\begin{tabular}{|c|c|c|c|}
\hline & Knaben & Mädchen & Summe \\
\hline Zangengeburt überhaupt. . . . . . . & $2,16 \mathrm{pCt}$. & 3,00 pCt. & $2,54 \mathrm{pCt}$. \\
\hline Erblichkeit allẹn $:$ & $\begin{array}{ll}0,16 \\
1,00, "\end{array}$ & $0,8 \overline{-}$ & $0,90 \%$ \\
\hline Trunksucht & $0,16 \%$ & 0,60 & 0,36 \\
\hline 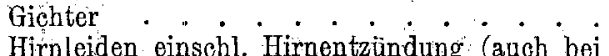 & $1,50 \%$ & 1,60 & 1,54, \\
\hline Infektionskrankheiten) & $0,67 \ldots$ & 1,40 & 1,00 \\
\hline Anderweitige Kopfverletzungen... & 0,66 & 0,20 & 0,45 \\
\hline Tuberkulose , . . . . & 0,16 & 0,20 & 0,18 \\
\hline Englische Krankheit . & - & 0,20 & 0,09 \\
\hline Seelische Schädigung . , . , , . & 0,16 & 0,20 & 0,18 \\
\hline
\end{tabular}

\begin{tabular}{|c|c|c|c|}
\hline & Knaben & Mädchen & Surme \\
\hline 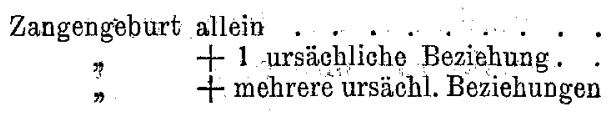 & $\begin{array}{l}0,16 \mathrm{pCt} . \\
0,50 \% \\
1,50 \%\end{array}$ & $\begin{array}{l}\overline{-}, 40 \mathrm{pCt} \\
2,60\end{array}$ & $\begin{array}{l}0,09 \mathrm{pCt} . \\
0,45 \\
2,00\end{array}$ \\
\hline
\end{tabular}

Wir sehen, dass die Zangengeburt als alleinige Ursache ganz verschwindend ist, dass vielmehr in $78 \mathrm{pCt}$. dieser Fälle, also in $3 / 4$, mehrere ursächliche Béziehungen zu der Zangengeburt treten. Daraus ergibt sich, dass ihre unmittelbare Einwirkung eine zweifelhafte ist. Dieser Eindruck verstärkt sich noch mehr, wenn wir auf einżelne Fälle năher eingehen wie z. B. Vater Trinker, Zangengeburt, Kopfverletzung: im 7. Jahre; $1 / 2$ Jahr später sollen zum ersten Male Anfälle aufgetreten sein - oder Vater nervös, Zangengeburt, Schädelverletzung mit Bewusstlosigkeit im 3. Jahre, Beginn der Anfälle im 6. Jahre - oder Zangengeburt, im 5. Jahre Hirnleiden mit linksseitiger Lähmung, darnach Anfälle. In derartigen Fällen frägt es sich, ob der Zangengeburt für die Entstehung der epileptischen Anfälle eine Bedeutung überhaupt beigelegt werden darf? Wenn man diese Frage bejaht, so kann ihr nur eine mittelbare Rolle zugestanden werden, insofern man annehmen will, dass die Ursache für die Entstehong der Zangengeburt und letztere selbst eine Schädigung des kindlichen Gehirns bedingt haben können. Diese Annahme ist in allen den Fällen berechtigter, in welchen wir eine nervöse erbliche Belastung verzeichnet finden, da wir 
wissen, dass durch sie eine angeborene Minderwertigkeit des Zentralnervensystems bedingt wird. In über der Hälfte der Fälle von Zangengeburt liegt erbliehe Belastung vor.

Wie schon beim Schwachsinn ausgeführt worden ist, stösst die Berechtigung der Eigenstellung der "Gichter" auf erhebliche Zweifel; in noch erhöhterem Masse trifft dies für die Epilepsie zu. Ueber letzteren Punkt liegt eine umfangreiche Literatur vor, welche einerseits die Gichter als Ausdrucksform der spasmophilen Diathese aufgefasst sehen will und die Ansicht vertritt, dass ein Uebergang in Epilepsie fast nie vorkommt. Auf der anderen Seite herrscht die Anschauung, dass zwischen-spasmophiler Diathese und Epilepsie nahe Beziehungen bestehen. Eine reinliche Scheidung und eine endgültige Klarstellung sind bis jetzt noch nicht erzielt worden. Mag man sich zu der Frage stellen, wie man will, so viel dürfte sicher sein, dass die spasmophile Diathese eine erhöhte Krampfbereitschaft des Gehirns im Gefolge hat und dass dementsprechend auch die Entwicklung der Epilepsie auf diesem Boden nichts Ungewöhnliches darstellen würde. Für unsere Zusammenstellung liegt. das Missliche darin, dass eine ärztliche Beobachtung der Gichter nur in den wenigsten Fällen vermerkt ist; dies wird auch in $\mathrm{Zu}$ kunft meist der Fall sein.

\section{Schwere Geburt.}

Die schwere Geburt wird in $2,45 \mathrm{pCt}$. zu der Entstehung der kindlichen Epilepsie in Beziehung gebracht, in 0,27 pCt. als alleinige Ursache. Bezüglich ihrer Schädigung des kindlichen Gehirns wird auf das beim Schwachsinn Gesagte hingewiesen, wonach eine lange dauernde schwere Geburt eine erheblichere Schädigung bedingt, als in der Mehrzahl der Fälle eine instrumentelle Entbindung.

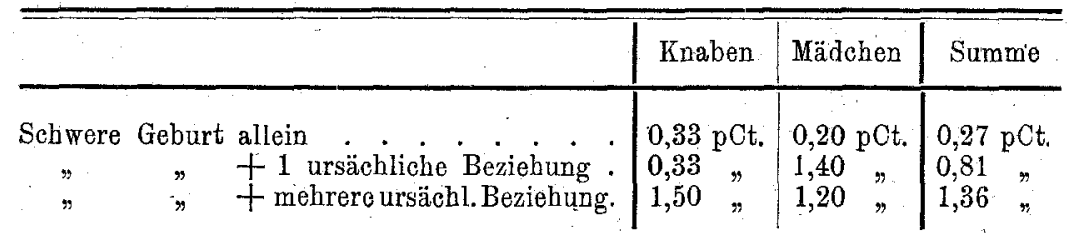

Es ergibt sich, dass bei über der Hälfte der Fälle neben der schweren Geburt noch mehrere ursächliche Beziehungen in Frage kommen. 
Schwere Geburt im Verein mit anderen ursächlichen Beziehungen.

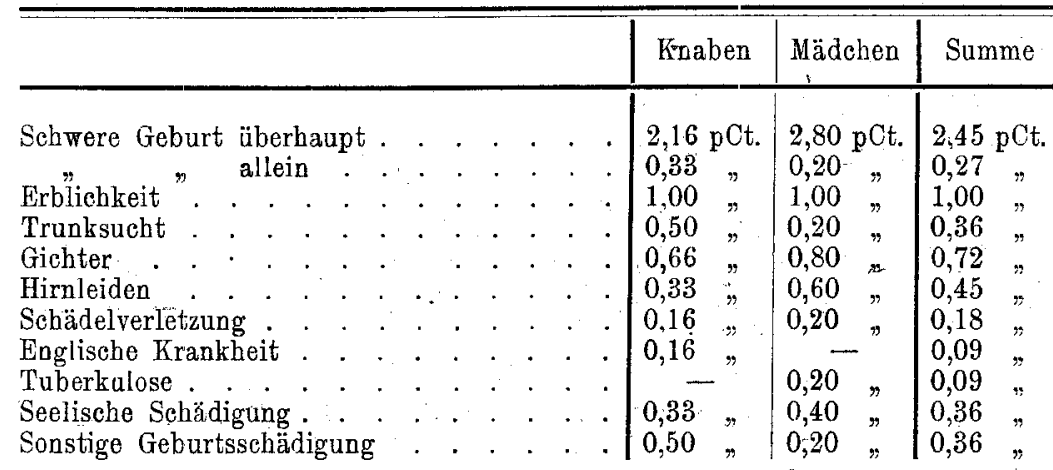

Erbliche Belastung liegt in über der Hälfte der. Fälle vor, wobei die Epilepsie der Erzeuger sehr vorherrscht. Nur in einem Falle wird die Epilepsie als angeboren bezeichnet; in 3 Fällen traten im Laufe des ersten Lebensjahres die epileptischen Erscheinungen auf. Der Zusammenhang zwischen schwerer Geburt und Epilepsie ist: vielfach ein sehr mittelbarer, wobei der schweren Geburt höchstens die Rolle eines vorbereitenden Momentes zukommt. Aehnlich verhält es sich bei den anderen Geburtsstörungen; wie wir noch sehen werden.

\section{Asphyktische Geburt.}

Soheintot geboren sind 1,45 pCt. der 1100 epileptischen Kinder; nur in $0,27 \mathrm{pCt}$. ist dieser Umstand der einzige ursächliche.

\begin{tabular}{|c|c|c|c|}
\hline & Knaben & Mädchen & Summe \\
\hline 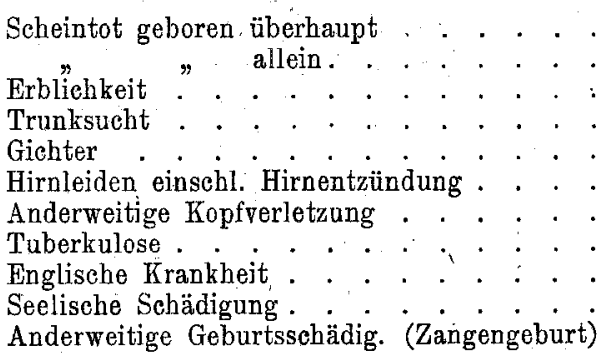 & $\begin{array}{l}1,33 \mathrm{pCt} \\
0,16 " \\
0,50 " \\
0,66 \\
0,16 " \\
\text { _ } " \\
0,16 \\
\text { - } "\end{array}$ & $\begin{array}{l}1,60 \mathrm{pCt} \\
0,40 " \\
0,60 " \\
0,40 \\
1,00 " \\
0,20 " \\
0,20 " \\
- \\
0,60\end{array}$ & $\begin{array}{l}1,45 \mathrm{pCt} \\
0,27 \\
0,54 " \\
0,54 " \\
0,54 " \\
0,09 " \\
0,09 " \\
0,09 " \\
0,27\end{array}$ \\
\hline & Knaben & Mädchen & Summe \\
\hline $\begin{array}{c}\text { Scheintot geboren allein . } \\
" \quad "+1 \text { ursächliche Beziehung } \\
" \quad \text { + mehrere ursächliche Be- } \\
\text { ziehungen . . . . }\end{array}$ & $\begin{array}{l}0,16 \mathrm{pCt} . \\
0,50 \% \\
0,66\end{array}$ & $\begin{array}{l}0,40 \mathrm{pCt} \\
0,20 \% \\
1,00\end{array}$ & $\begin{array}{l}0,27 \mathrm{pCt} . \\
0,36 " \\
0,81\end{array}$ \\
\hline
\end{tabular}


Auffällig ist die verhältnismässig geringe erbliche Belastung, insbesondere das völlige Fehlen der Trunksucht in der Aszendenz. Gichter und Hirnleiden finden wir je fast in der Hälfte der Fälle. Ein nennenswerter Unterschied der Geschlechter liegt nicht vor.

\section{Frühgeburt.}

Frühgeburt finden wir in keinem der Fälle als alleinige Ursache aufgeführt. Die Beteiligung der beiden Geschlechter ist genau dieselbe und beträgt 1 pCt.

Frühgeburt im Verein mit anderen ursächlichen Beziehungen.

\begin{tabular}{|c|c|c|c|}
\hline & Knaben & Mädchen & Summe \\
\hline 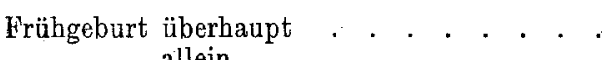 & $1,00 \mathrm{pCt}$ & $1,00 \mathrm{pCt}$. & $1,00 \mathrm{pCt}$. \\
\hline \multirow[t]{2}{*}{ 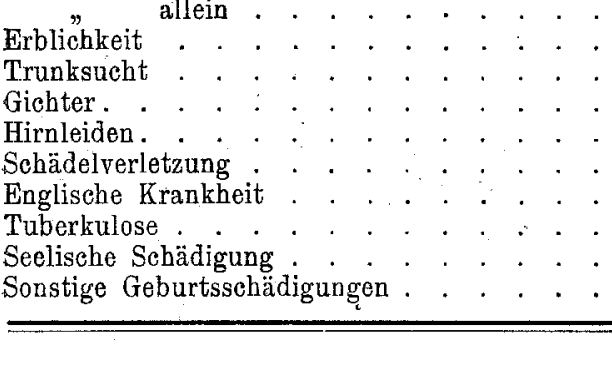 } & $\begin{array}{r}0,33 \\
0,16 " \\
0,50 " \\
= \\
= \\
0,16 \\
-\end{array}$ & 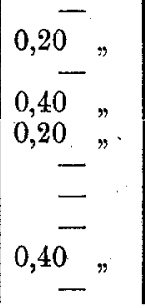 & $\begin{array}{r}0,27 \\
0,09 \\
0,18= \\
= \\
\overline{-} \\
0,27 \\
-\end{array}$ \\
\hline & Knaben & Mädchen & Summe \\
\hline $\begin{array}{l}\text { trühgeburt allein } \\
\qquad \begin{array}{l}+1 \text { ursäohliche Beziehung } \\
\quad+\text { mehrere ursächl. Beziehungen }\end{array}\end{array}$ & $\begin{array}{l}0,50 \mathrm{pCt} \\
0,50\end{array}$ & $\begin{array}{l}0,80 \mathrm{pCt} \\
0,20\end{array}$ & $\begin{array}{l}0,6 \bar{p} \mathrm{Ct} . \\
0,36\end{array}$ \\
\hline
\end{tabular}

In $3 / 4$ der Fälle erfolgte die Frühgeburt im 7. Monat, einmal im 8. Monat. Beim Rest war der Zeitpunkt der Frühgeburt nicht angegeben. In etwas mehr als der Hälfte lag erbliche Belastung vor.

Nur in einem Falle wurde die Epilepsie als angeboren bezeichnet; im 1. Jahre trat sie in mehr als einem Drittel der Fälle auf. Bei der Frühgeburt finden wir ebenfalls, dass der Zusammenhang mit der Epilepsie ein loser ist. Für die Annahme, dass die Frühgeburt ein weniger widerstandsfähiges Nervensystem im Gefolge hat, scheint der Umstand zu sprechen, dass in $1 / 3$ der Fälle nach einer seelischen Schädigung sich die Anfälle eingestellt haben.

\section{Zwillingsgeburt.}

In keinem Falle bildet sie die alleinige Ursache der Epilepsie; ihr Vorkommen beträgt 0,90 pCt. Auch hier ist in der Vorgeschichte Trunksucht nicht erwähnt. 
Zwillingsgeburt im Verein mit anderen ursächlichen Beziehungen.

\begin{tabular}{|c|c|c|c|}
\hline & Knaben & Mädchen & Summe \\
\hline 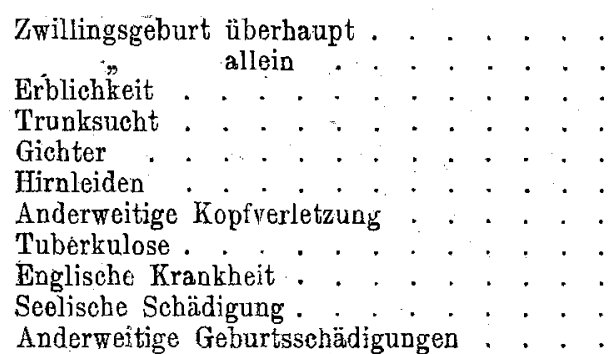 & $\begin{array}{l}1,00 \mathrm{pCt} . \\
0,50 \\
0,16 " \\
0,33= \\
0,16 " \\
\text { - } " \\
0,16 "\end{array}$ & $\begin{array}{c}0,80 \mathrm{pCt} \\
\text { - } \\
\overline{-} \\
0,20 \\
0,40 " \\
0,20 " \\
\text { - } \\
=\end{array}$ & $\begin{array}{l}0,90 \mathrm{pCt} . \\
0,27 \\
0,18 \\
0,36 " \\
0,18= \\
0,09\end{array}$ \\
\hline & Knaben & Mädchen & Summe \\
\hline $\begin{array}{cc}\text { Zwillingsgeburt allein } \\
\Rightarrow \quad+1 \text { ursächliche Beziehung } \\
\Rightarrow & + \text { mehrere ursächl. Beziehung. }\end{array}$ & $\begin{array}{l}-\overline{\mathrm{p}} \mathrm{Ct} . \\
0,50 \mathrm{p}\end{array}$ & $0,80 \mathrm{pCt}$ & $\begin{array}{l}0,63 \mathrm{pCt} \\
0,27\end{array}$ \\
\hline
\end{tabular}

Erbliche Belastung finden wir in $1 / 3$ vor; Hirnleiden in fast der Hälfte. In keinem Falle wird die Epilepsie als angeboren bezeichnet; nùr in einem Falle traten im Laufe des ersten Lebensjahres epileptische Anfälle auf.

Für die Beziehung der Zwillingsgeburt zur Epilepsie gilt das bei der Frühgeburt Gesagte; sie schafft eben oine erhöhte Anfälligkeit des kindlichen Gehirns.

\section{Nabelblutung.}

Nabelblutung wird nur in einem weiblichen Falle und zwar im Verein mit anderen ursächlichen Beziehungen aufgeführt $(0,09$ pCt. der Gesamtzahl): Der Vater dieses epileptischen Mädchens ist an Tuberkulose gestorben; während des Zahnens sollen heftige Gichter aufgetreten sein und danach epileptische Anfälle.

\section{Zusammenfassung.}

Bei der Entstehung der kindlichen Epilepsie kommt den Geburtsschädigungen mit 1,09 pCt. als alleiniger Ursache eine untergeordnete Bedeutung zu. Erwähnung finden sie in 8,54 pCt. In mindestens einem Drittel dieser Fälle liegt erbliche Belastung vor, über deren Beschaffenheit. noch Näheres berichtet werden wird. Bei der überwiegenden Zahl der Fälle stellt die Geburtsschä̀digung eine die spätere Entwicklung der Epilepsie begünstigende ursächliche Beziehung dar und beansprucht als solche unsere Aufmerk- 
samkeit und Rücksichtnahme. Im Interesse der Bekämpfung der Epilepsie ist es auch gelegen, wenn die Geburtsschädigungen nach Möglichkeit ausgeschaltet werden. Warum durch eine Geburtsschädigung in dem einen Falle Schwachsinn, in dem anderen Epilepsie hervorgerufen wird, lässt sich vielfach nicht klarstellen. Die beim Schwachsinn besprochenen pathologisch-anatomischen Vorgänge und Veränderungen sind im wesentlichen auch für die Epilepsie zutreffend.

Vogt (1) warnt vor Ueberschätzung der Geburtsschädigung. „In Betracht können nur besonders schwere Traumata kommen, namentlich unter dem Geburtsakt, aber auch diese nicht allein."

Nach Ranke (2) ist es wahrscheinlich, dass, um ein Trauma wirklich zu einem verderblichen zu machen, noch ein in der Organisation 'des Gehirns' selbst gegebenes Moment hinzukommen muss. "Nur auf einem a priori durch Krankheit oder durch mangëlhafte Entwicklung geschwächten Boden können so schwere Traumafolgen erwachsen."

Binswanger (3) unterscheidet zwischen ererbter, intrauterin erworbener und während des extrauterinen Individuallebens erworbener Prädisposition. Bezüglich der traumatischen Schädigungen des Schädels und Gehirns während des Geburtsakts äussert sich Binswanger (3), wie folgt: „Die Fälle sind besonders bemerkenswert; bei denen gröbere Verletzungen des Schädels oder des Gehirns mit sichtbaren Folgen (z. B. Meningitiden, Enzephalitiden, Blutungen mit nachfolgenden Kinderlähmungen) des Traumas nicht rorhanden-sind. Es handelt sich dann vorwaltend um Schädelkompression bei forciertem Forceps oder bei Wendungen oder um Hirnerschütterungen durch Sturz auf den Kopf bei präzipitierten Geburten, die scheinbar ohne alle nachteiligen Folgen für die geistige und körperliche Entwicklung des Kindes sein können." Auch ohne grobe materielle Verletzung kann das Trauma nach Vogt (1) vorübergehende oder dauernde Aenderungen der zentralen Innervationsvorgänge hervorbringen. Ahlfeld (4) verhält sich nicht ganz ablehnend, wenn er sagt: "Die Annahme, dass Kinder, die mit der Zange entwickelt wurden, häufig in ihrem weiteren Leben an psychischen Störungen und Epilepsie leiden sollten, ist noch nicht bewiesen, aber durch einzelne Beobachtungen nicht unwahrscheinlich gemacht." Gewissen Umständen hat man nach Féré (6) die Schuld für die Entstehung der kindlichen Epi- 
lepsie beigemessen. "Ob diese Dinge wirklich die ihnen zugeteilte Rolle spielen, ist schwer zu sagen; aber sie verdienen, genannt zu werden."

Eine erworbene Prädisposition des Individuums und damit eine gesteigerte Verletzbarkeit des Zentralnervensystems liegt nach Binswanger (3) auch in jenen Fällen vor, in welchen traumatische Schädigungen des Schädels bzw. des Gehirns während des Geburtsaktes die Grundlage einer dauernden Beeinträchtigung des Nervensystems bilden.

Volland (5) hat bei 1500 Fällen in $45=3$ pCt. Geburtsstörungen verzeichnet gefunden, 13 dieser 45 Fälle weisen erbliche Belastung auf. Es ist nach Volland (5) einleuchtend, dass beim Zusammentreffen der beiden. Umstände eine gesteigerte Prädisposition zur Erkrankung des Individuums vorliegt.

Volland (5) , kommt zu dem Ergebnis: Im Vergleich zu der grossen Häufigkeit der Epilepsie spielen bei ihrer Entstehung die Geburtsstörungen nur eine untergeordnete Rolle. In einer kleinen Anzahl von Fällen sind die Geburtsstörungen als vorbereitende Ursache für die spätere Epilepsie anzuschuldigen. „Auch für den Fall des Zutreffens einer Geburtsanomalie als ätiologisch mögliches Moment kann doch der Hauptgrund in anderen Ursachen liegen [Belastung, namentlich Potus des Vaters, Infektionskrankheiten, Trauma" ( $\mathrm{V} \circ \mathrm{gt})]$.

Wir sehen aus diesen Literaturangaben, dass auch die früheren Autoren in der Bewertung der Geburtsschädigung für die Entstehung der kindlichen Epilepsie recht zurückhaltend gewesen sind.

Vergleichen wir den Schwachsinn mit der Epilepsie, so finden wir, dass die Geburtsschädigung als alleinige Ursache beim Schwachsinn häufiger genannt ist, als bei der Epilepsie und zwar im Verhältnis von 2,81 pCt: 1,09 pCt., also über doppelt so häufig. Beim Schwachsinn finden wir Geburtssehädigungen überhaupt in 13,63 pCt., bei der Epilepsie nur in 8,54 pCt. aufgeführt, also über $1 / 3$ weniger.

Nicht ohne Interesse ist auch eine vergleichsweise Uebersicht der erblichen Belastung der Geburtsschädigungen beim Schwachsinn und bei Epilepsie; sie ergibt, dass bei dem Schwachsinn die erbliche Belastung mit Schwachsinn, bei der Epilepsie die mit Epilepsie deutlich überwiegt. Der mütterliche Anteil herrscht mit Ausnahme der Trunksucht vor. Die erbliche Belastung betrug im ganzen rund $50 \mathrm{pCt}$. 
Unsere Betrachtung bestätigt die auch anderweitig gemachte Erfahrung, dass sowohl bei der Entstehung des kindlichen Schwachsinns als auch der kindlichen Epilepsie meist mehrere ursächliche Beziehungen in Frage kommen, welche teils eine vorbereitende, teils eine auslösende Rolle spielen. In diesem Sinne haben wir auch die Geburtsschädigungen kennen gelernt.

\section{Literatur.}

1. Vogt, Die Epilepsie im Kindesalter.

2. Ranke, Vortrag. Naturforscherversamml. Strassburg 1885.

3. Binswanger, Die Epilepsie. 1913, 2. Aufl.

4. A blfeld, Lehrb. d. Geburtsh. 1898.

5. Volland, Allg. Zeitschr. f. Psych. Bd. 63. S. $725 \mathrm{ff}$.

6. Féré, Die Epilepsie. 1899. 